\title{
LIE ALGEBRAS OF DIFFERENTIAL OPERATORS IN TWO COMPLEX VARIABLES
}

\author{
By Artemio González-López, Niky Kamran, $\dagger$ and Peter J. Olver $\ddagger$
}

1. Introduction. Of the problems that occupied the researchers working in the area of Lie algebras at the turn of the last century, one can identify at least two which led to important developments which are still of great interest today. One is the classification problem for abstract finite-dimensional complex semisimple Lie algebras, which was solved by Elie Cartan in his thesis, [7]. The other problem, which is different in nature, but equally interesting, is that of classifying, up to local diffeomorphisms, the finite-dimensional Lie algebras of vector fields $\mathbf{v}=$ $\Sigma \xi^{i}(x) \partial / \partial x^{i}$ defined on an open subset of the complex Euclidean space $\mathbb{C}^{n}$. For nonsingular Lie algebras (i.e. those of locally constant dimension), this problem was solved by Lie in the cases $n=1$ and 2, [14]; Lie also claimed to have solved the case $n=3$, but only an incomplete classification ever appeared in print, [15, Chapter 2].

One may also consider the more general question of classifying the finite-dimensional Lie algebras of first order differential operators $\mathscr{O}=$ $\Sigma \xi^{i}(x) \partial / \partial x^{i}+f(x)$. These Lie algebras appear naturally in the theory of projective (or multiplier) representations of Lie groups, [18]. They are also relevant in quantum mechanics through the so-called algebraic approach to scattering theory and molecular dynamics, [3]. In this latter context, R. Levine, [13], posed the problem of classifying all the second order time-independent Schrödinger operators which lie in the universal enveloping algebra of a finite dimensional Lie algebra $g$ of first order differential operators, i.e. which can be written as a constant coefficient bilinear combination of the generators of $\mathrm{g}$. This problem is also a generalization of the factorization problem for differential operators, [20].

\footnotetext{
Manuscript received 8 December 1989; revised October 1990. $†$ Research supported in part by an NSERC Grant. $\ddagger$ Research supported in part by NSF Grant DMS 89-01600. American Journal of Mathematics 114 (1992), 1163-1185
} 
In an earlier pair of papers, [11], [12], (see also [22]), two of the present authors gave a complete solution to Levine's problem on the line, that is in the case $n=1$. The basic approach, which in principle extends to all dimensions, consists of the following two steps. First, one classifies all finite-dimensional Lie algebras of differential operators up to local diffeomorphisms, leading to a complete set of normal forms for these Lie algebras. Then one solves the equivalence problem for second order differential operators, thereby determining when a given Schrödinger operator can be written in bilinear form using one of the Lie algebras obtained in the first step. The resulting potentials, named Lie algebraic potentials in reference 12 , include many of the well-known one-dimensional potentials of quantum mechanics, as well as a number of previously unstudied ones. A significant omission is the Coulomb potential, which is known to be expressible as a bilinear combination of planar differential operators generating a finite-dimensional Lie algebra, [3], but cannot be so expressed using purely one-dimensional operators. This fact, and the general problem of determining new and interesting classes of potentials for which the Schrödinger equation is amenable to treatment by the algebraic method of Levine $e t$ al. motivate the present work.

In this paper we give a complete solution to the classification problem for finite dimensional nonsingular complex Lie algebras of first order differential operators in the planar case $n=2$, exhibiting a complete list of such Lie algebras. This corresponds to the first of the two steps mentioned above for the solution to Levine's problem in $\mathbb{C}^{2}$.

There are three basic steps required to classify finite dimensional Lie algebras of differential operators over a given manifold. First, one needs to classify the finite dimensional Lie algebras of vector fields $\mathfrak{h}$ on the manifold up to diffeomorphism. Secondly, for each of these Lie algebras, one needs to classify all possible finite dimensional $\mathfrak{h}$-modules $m$ of $C^{\infty}$ functions. Finally, for each of the modules $m$, one needs to determine the first cohomology space $H^{1}\left(\mathfrak{h}, C^{\infty}(M) / m\right)$ of the Lie algebra $\mathfrak{h}$ with coefficients in the quotient $\mathfrak{h}$-module $C^{\infty}(M) / m$. As detailed in Section 2 , this is equivalent to effecting the required classification.

In the case of $\mathbb{C}^{2}$, the first step in our classification procedure was already achieved by Lie, [14], who obtained 24 essentially different classes of nonsingular Lie algebras of vector fields, some of which depend on parameters. (See [2] for an English translation of Lie's fundamental paper.) A nice summary of the basic classification appears in 
the "Gruppenregister" at the end of volume 5 of Lie's collected works, [16]. See also Bianchi, [5; Chapter 11], and Campbell, [6; Chapters 21, 22], for other treatments of Lie's result. Our paper is devoted to the two subsequent stages of the classification, namely the determination of the finite dimensional modules of smooth functions for the Lie algebras on Lie's list, and the corresponding cohomology spaces.

We should point out that there are several features of the twodimensional case which were absent in the much simpler one-dimensional case, and which make our problem more interesting. The Lie algebras of vector fields in $\mathbb{C}^{2}$ can have arbitrarily high dimension, the module may depend on arbitrary functions, the polynomial modules need not be spanned by monomials, and the structure of the modules may depend critically on the value of the essential parameter on which these Lie algebras depend (even to the extent of being different for rational and irrational values of the parameter). As a consequence, the expression for the Lie algebra cohomology may be extremely complicated. Finally, we should remark that $H^{1}\left(\mathfrak{h}, C^{\infty}(M) / m\right)$ need not vanish if $\mathfrak{h}$ is semisimple as suggested by the Whitehead lemma, cf. [10], since $C^{\infty}(M) / m$ is not a finite dimensional module.

Extensions of this work to higher dimensions are readily apparent, but the computational difficulties are formidable. A more modest program would be to classify the Lie algebras of differential operators corresponding to transitive, primitive transformation groups, which are completely known in all dimensions, [8]. These are of interest for several reasons, including their connections with nonlinear ordinary differential equations with superposition principles, [21]. Also, the extensions of this classification to real two dimensional spaces can be done, since we now have, [9], a complete classification of the real Lie algebras of vector fields on $\mathbb{R}^{2}$.

We would like to thank Willard Miller, Jr. for many insightful comments on this work. Also, we wish to thank Thomas Hawkins for sharing his research into the history of Lie's classification of Lie algebras of vector fields. The support and hospitality of the Institute for Mathematics and its Applications (I.M.A.) is gratefully acknowledged.

2. Lie algebras of differential operators - general facts. Let $M$ be a smooth manifold. Consider the space $\mathbb{D}=\mathbb{D}(M)$ of all first order differential operators on $M$. In local coordinates $\mathbf{x}=\left(x_{1}, \ldots, x_{n}\right)$, these take the form 


$$
\mathscr{Q}=\sum_{i=1}^{n} f_{i}(\mathbf{x}) D_{i}+g(\mathbf{x})=\mathbf{f}(\mathbf{x}) \cdot \mathbf{D}+g(\mathbf{x}),
$$

where $D_{i}$ denotes derivative with respect to $x_{i}$. There is a natural Lie bracket which makes $\mathbb{D}$ into a Lie algebra, given by the commutator $[\mathscr{O}, \delta]=\mathscr{O} \cdot \varepsilon-\delta \cdot \mathscr{O}$. The fundamental problem to be addressed in this paper is the determination of all possible finite-dimensional Lie subalgebras of the Lie algebra $\mathbb{D}$.

There are two natural classes of coordinate changes which act on the algebra of differential operators. The first are the (invertible) smooth changes of variables: $\overline{\mathbf{x}}=\varphi(\mathbf{x})$, which transform the operator (2.1) according to the standard chain rule formula. Second, we can rescale the field variable by smooth functions $\psi(\mathbf{x})$, which, in order to preserve the Lie algebra structure of $\mathbb{D}$, must act on differential operators according to

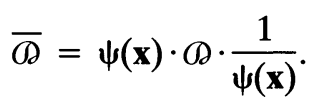

We will call two (Lie algebras of) differential operators equivalent if there is a change of variables $\overline{\mathbf{x}}=\varphi(\mathbf{x})$ and a scalar-valued function $\psi(\mathbf{x})$ such they are related by (2.2).

Let $\mathbb{C} \mathbb{D}$ denote the subalgebra of all vector fields $\mathbf{v}=\mathbf{f}(\mathbf{x}) \cdot \mathbf{D}$ on $M$. Let $\mathbb{M} \subset \mathbb{D}$ denote the abelian subalgebra of multiplication operators, consisting of the operators (2.1) with $\mathbf{f} \equiv 0$, so that $\mathbb{M} \cong C^{\infty}(M)$. The vector fields act naturally on the multiplication operators, giving $\mathbb{D}$ $=\mathbb{V} \ltimes \mathbb{M}$ the structure of a semidirect product. Let $\pi: \mathbb{D} \rightarrow \mathbb{V}$ denote the projection $\pi(\mathscr{O})=\mathbf{f} \cdot \mathbf{D}$ for $\mathscr{O}=\mathbf{f} \cdot \mathbf{D}+g$. Then the above notion of equivalence preserves the exact sequence of Lie algebra homomorphisms

$$
0 \rightarrow \mathbb{M} \rightarrow \mathbb{D} \stackrel{\pi}{\rightarrow} \mathbb{V} \rightarrow 0
$$

Any finite-dimensional Lie subalgebra $\mathfrak{g} \subset \mathbb{D}$ is spanned by differential operators

$$
\mathbf{v}_{1}+f_{1}(\mathbf{x}), \ldots, \mathbf{v}_{r}+f_{r}(\mathbf{x}), h_{1}(\mathbf{x}), \ldots, h_{m}(\mathbf{x})
$$


where $\mathbf{v}_{1}, \ldots, \mathbf{v}_{r}$ are linearly independent vector fields spanning an $r$ dimensional Lie algebra $\mathfrak{h}=\pi(\mathfrak{g})$ of vector fields, and the functions $h_{1}(\mathbf{x}), \ldots, h_{m}(\mathbf{x})$ span the abelian subalgebra $m=g \cap \mathbb{M}$ of multiplication operators in $\mathfrak{g}$. Thus $\mathfrak{g}$ also has the structure of a semidirect product: $\mathfrak{g} \cong \mathfrak{h} \ltimes m$. There is a convenient interpretation of this data in cohomological terms. (See Miller, [18], for a slightly different version of these results.) In general, if $\mathfrak{h}$ is a Lie algebra, and $\mathbf{M}$ an $\mathfrak{h}$-module, the space of $i$-cochains, $i \geq 0$, is the $\mathfrak{h}$-module $C^{i}(\mathfrak{h}, \mathbf{M})$ consisting of all alternating $i$-linear maps $F: \mathfrak{h} \times \cdots \times \mathfrak{h} \rightarrow \mathbf{M}$. Let $\delta: C^{i}(\mathfrak{h}, \mathbf{M}) \rightarrow$ $C^{i+1}(\mathfrak{h}, \mathbf{M})$ be the usual coboundary operator, cf. [10]. The space of $i$-cocycles is $Z^{i}(\mathfrak{h}, \mathbf{M})=\operatorname{Ker} \delta \cap C^{i}(\mathfrak{h}, \mathbf{M})$, the space of $i$-coboundaries is $B^{i}(\mathfrak{h}, \mathbf{M})=\delta\left[C^{i-1}(\mathfrak{h}, \mathbf{M})\right]$, and $H^{i}(\mathfrak{h}, \mathbf{M})=Z^{i}(\mathfrak{h}, \mathbf{M}) / B^{i}(\mathfrak{h}, \mathbf{M})$ is the $i^{\text {th }}$ cohomology space.

Theorem 1. Let $M$ be a smooth manifold, and $C^{\infty}(M)$ the algebra of smooth scalar-valued functions on $M$. There is a one-to-one correspondence between finite dimensional Lie algebras of differential operators on $M$ and triples $(\mathfrak{h}, m, \mathbf{F})$, where

1. $\mathfrak{h}$ is a finite-dimensional Lie algebra of vector fields on $M$,

2. $m \subset C^{\infty}(M)$ is a finite-dimensional $\mathfrak{h}$-module of functions on $M$,

3. $\mathbf{F} \in Z^{1}\left(\mathfrak{h}, C^{\infty}(M) / m\right)$ is a 1-cocycle with coefficients in the quotient module $C^{\infty}(M) / m$.

Proof. Consider the Lie algebra $g$ spanned by the differential operators (2.3), and let $\mathfrak{h}$ and $m$ be defined as above. Since $\left[\mathbf{v}_{i}+f_{i}, h_{j}\right]$ $=\mathbf{v}_{i}\left(h_{j}\right)$ must also lie in $g$, and hence in $m$, we see that $m$ must be an $\mathfrak{h}$-module, in accordance with condition 2 . Next, define the 1-cochain $\mathbf{F}: \mathfrak{h} \rightarrow C^{\infty}(M)$ by $\left\langle\mathbf{F} ; \mathbf{v}_{i}\right\rangle=f_{i}$, and extend by linearity. The map

$$
D_{\mathbf{v}}=\mathbf{v}+\langle\mathbf{F} ; \mathbf{v}\rangle
$$

associates a differential operator $D_{\mathfrak{v}} \in \mathfrak{g}$ with any vector field $\mathbf{v} \in \mathfrak{h}$. However, the functions $f_{i}$ are not uniquely determined since we can replace $f_{i}$ by $f_{i}+h$ for any $h \in m$ without changing $\mathrm{g}$. Thus, we should regard each function $f=\langle\mathbf{F} ; \mathbf{v}\rangle$ as lying in the quotient module $C^{\infty}(M) /$ $m$, and $\mathbf{F}$ is a well-defined $C^{\infty}(M) / m$-valued 1-cochain. Moreover, the commutator $\left[D_{v}, D_{w}\right]=D_{[v, w]}+\langle\delta \mathbf{F} ; \mathbf{v}, \mathbf{w}\rangle$ lies in $\mathfrak{g}$ if and only if $\langle\delta \mathbf{F} ; \mathbf{v}, \mathbf{w}\rangle \in \boldsymbol{m}$, which implies that $\mathfrak{g}$ is a Lie algebra if and only if $\mathbf{F}$ is 
a $C^{\infty}(M) / m$-valued 1-cocycle. Conversely, given a triple $(\mathfrak{h}, m, \mathbf{F})$ satisfying the hypotheses, the direct sum $\mathfrak{g}=\{\mathbf{v}+\langle\mathbf{F} ; \mathbf{v}\rangle \mid \mathbf{v} \in \mathfrak{h}\} \oplus m$ is easily shown to be a Lie algebra.

To classify Lie algebras of differential operators, we only need to determine representatives of different equivalence classes. The equivalence relation between differential operators will induce an equivalence relation between the corresponding triples $(\mathfrak{h}, m, \mathbf{F})$. First, the change of variables $\overline{\mathbf{x}}=\varphi(\mathbf{x})$ acts on the Lie algebra of vector fields $\mathfrak{h}$ in the obvious manner; it similarly transforms the module $m$ and the cocycle F. Moreover, for a fixed $\mathfrak{h}$, two $\mathfrak{h}$-modules are equivalent if they are mapped to each other under a change of variables in the isotropy group of $\mathfrak{h}$. Rescaling the field variable via (2.2) does not affect $\mathfrak{h}$ or $m$, but has the effect of replacing the differential operator $D_{v}$ in (2.4) by the operator $D_{\mathbf{v}}-\mathbf{v}(\log \psi)=D_{\mathbf{v}}-\langle\delta \log \psi ; \mathbf{v}\rangle$, which amounts to subtracting the coboundary $\delta \log \psi$ from the 1-cocycle F. Also, since the coboundary operation preserves $m$, we can regard $\log \psi$ as lying in $C^{\infty}(M) / m$. Therefore, for fixed $\mathfrak{h}$ and $m$, two cocycles $\mathbf{F}$ and $\tilde{\mathbf{F}}$ will determine equivalent Lie algebras of differential operators if and only if they are cohomologous. In this way the different Lie algebras of differential operators are classified by cohomology classes in $H^{1}\left(\mathfrak{h}, C^{\infty}(M) / m\right)$.

Theorem 2. Let M be a smooth manifold. There is a one-to-one correspondence between equivalence classes of finite dimensional Lie algebras of differential operators on $M$ and equivalence classes of triples $(\mathfrak{h}, m,[\mathbf{F}])$, where

1. $\mathfrak{h}$ is a finite-dimensional Lie algebra of vector fields on $M$,

2. $m \subset C^{\infty}(M)$ is a finite-dimensional $\mathfrak{h}$-module of functions on $M$,

3. $[\mathbf{F}] \in H^{1}\left(\mathfrak{h}, C^{\infty}(M) / m\right)$.

Two such triples are equivalent if they are directly mapped to each other by a change of variables, the cohomology taking care of the rescaling (2.2). We will always work with a specific representative of each equivalence class $[\mathfrak{h}, m,[\mathbf{F}]]$. Moreover, in most cases, the isotropy group is trivial, so we can ignore the equivalence of modules under isotropy. There are thus three steps in to the general classification procedure for finite-dimensional Lie algebras of differential operators: 1 . classify finite-dimensional Lie algebras $\mathfrak{h}$ of vector fields on $M$ up to change of variables; 2 . classify finite-dimensional $\mathfrak{h}$-modules $m ; 3$. determine the 
cohomology space $H^{1}\left(\mathfrak{h}, C^{\infty}(M) / m\right)$. Our solution to these problems is summarized in Tables 1-3. In this paper, we will be only concerned with the local classification of nonsingular (i.e. generic) Lie algebras of differential operators. Analysis of singular points, where the dimension reduces, is considerably more complicated, even for the case of a single vector field. (See the discussion of normal forms given in Arnol'd, [4; Chapter 5].) In particular, we will work entirely in local coordinates, and ignore global topological questions.

3. Classification of modules. We now specialize the preceding considerations to the case when $M$ is an open subset of $\mathbb{C}^{2}$. We use the notation

$$
\mathscr{O}=f_{1}(x, y) p+f_{2}(x, y) q+g(x, y),
$$

for a differential operator in the plane, where we abbreviate $p=D_{x}$, $q=D_{y}$. Lie's classification of finite-dimensional Lie algebras of vector fields on $\mathbb{C}^{2}$ is summarized in Table 1 . (We have omitted the trivial case when the Lie algebra just consists of the 0 vector field.) The first column gives our number for the (class of) Lie algebras, and the second column gives a basis. Some of these algebras have been written in slightly more convenient coordinate systems than are to be found in Lie. The third column gives its structure as an abstract Lie algebra. Here, $\mathfrak{h}_{2}=\mathbb{C} \ltimes$ $\mathbb{C}$ denotes the unique solvable two-dimensional Lie algebra. The last column indicates where the Lie algebra lies in Lie's "Gruppenregister," [16]. The list is not completely duplication-free (see Campbell, [6]). For example, the Lie algebra of type 5 for a given $0 \neq \alpha \in \mathbb{C}$ is clearly isomorphic to the same algebra with constant $1 / \alpha$ by interchanging $x$ and $y$; also type 4 is a special case of type 18 , although it is convenient to treat this case separately. Note that the transitive, primitive Lie algebras in the plane are cases 7,8 and 15 , i.e. the special linear affine group, the general linear affine group, and the full projective group in the plane.

The second step in the classification procedure is to determine the most general finite-dimensional $\mathfrak{h}$-module of functions for each Lie algebra $\mathfrak{h}$ appearing in Table 1 . These are listed in Table 2 , which we discuss in detail below, omitting the fairly straightforward proofs in order to keep the exposition short. To begin with, a module $m$ is trivial, written $m=0$, if it consists of the zero function alone. The next simplest case 
is when $m$ consists of just the constant functions, which we write $m=$ $\{1\}$, since the function 1 generates $m$. Every Lie algebra of vector fields admits either of these two possibilities as modules. In some cases (11, $15,23,24)$ these are the only possibilities, but usually there' will be other possible types of finite-dimensional modules.

Definition 3. An $x$-translation module is a finite-dimensional module for the one-dimensional Lie algebra generated by $p=\partial_{x}$. A semipolynomial $x$-translation module is a finite-dimensional module spanned by functions $f_{k}(x, y)=\Sigma_{i} g_{k}^{i}(y) x^{i}$ and all their $x$ derivatives $\partial^{j} f_{k} / \partial x^{j}$, $j \geq 0$, where $g_{k}^{i}(y)$ are functions of $y$. If the $g_{k}^{i}(y)$ are themselves polynomials, the module is called a polynomial $x$-translation module. If the module is spanned by semimonomials $g_{k}(y) x^{i}$ and their $x$ derivatives, then the module is called a semimonomial $x$-translation module. If the $g_{k}$ 's are monomials, so there is a basis consisting of monomials $x^{i} y^{j}$, then we have a monomial $x$-translation module.

Proposition 4. Every $x$-translation module is a direct sum of finitely many submodules $m_{\lambda}=\hat{m}_{\lambda} e^{\lambda x}$, where $\hat{m}_{\lambda}$ is a semi-polynomial $x$-translation module.

It is interesting to contrast the situation here with the corresponding one-dimensional version, where $\mathfrak{h}$ is generated by $p=\partial_{x}$, but the functions are only allowed to depend on $x$. In that case, [12], every translation module is spanned by monomials $x^{i} e^{\lambda x}$. In contrast, by admitting $y$ dependence, we no longer retain the property that translation modules are spanned by monomials. This complicating fact will cause a number of difficulties in our classification procedure.

Definition 5. A translation module is a finite-dimensional module for the Lie algebra $\{p, q\}$ of translations in the plane. The space spanned by a finite collection of polynomials $f_{k}(x, y)=\sum_{i, j} a_{i j} x^{i} y^{j}$, and all their $x$ and $y$ derivatives $\partial^{m} f_{k} / \partial x^{l} \partial y^{m-l}, m \geq l \geq 0$, is called a polynomial translation module. If the module is spanned by monomials, $x^{i} y^{j}$, then it is called a monomial translation module.

Again, it is perhaps surprising that not every polynomial translation module is spanned by monomials. An example is the polynomial translation module spanned by $x^{2}+y, x, 1$. In the special case of a translation module generated by monomials $x^{i} y^{j}$, the exponents $(i, j)$ lie in some subset $\mathbf{S} \subset \mathbb{Z}^{+} \times \mathbb{Z}^{+}$. Note that if $(i, j) \in \mathbf{S}$, and $0 \leq i^{\prime}<i, 0 \leq j^{\prime}<$ $j$, then $\left(i^{\prime}, j^{\prime}\right) \in \mathbf{S}$, so $\mathbf{S}$ is a (Young) diagram (or Ferrers graph), [17]. 
We remark that any nonmonomial translation module can always be included in a larger monomial module merely by taking all the constituent monomials of the elements.

Proposition 6. Every translation module is a direct sum of finitely many submodules $m_{\lambda, \mu}=\hat{m}_{\lambda, \mu} e^{\lambda x+\mu y}$, where $\hat{m}_{\lambda, \mu}$ is a polynomial translation module.

This takes care of cases 1 and 4 in Table 2. Many of the other cases are handled by the following pair of simple lemmas.

Lemma 7. If $\mathfrak{h}$ contains the vector fields $p, q$, and $(a x+b y) p+$ $(c x+d y) q, a d-b c \neq 0$, then any finite dimensional $\mathfrak{h}$-module is a polynomial translation module.

Lemma 8. If $\mathfrak{h}$ contains the vector fields $p, q$, and

$$
\begin{gathered}
(a x+b y) p+(c x+d y) q, \quad \text { where } a d-b c \neq 0, \\
\left(\alpha x^{2}+\beta x y+\gamma y^{2}\right) p+\left(\lambda x^{2}+\mu x y+v y^{2}\right) q, \\
\text { where rank }\left(\begin{array}{llll}
0 & \alpha & \beta & \gamma \\
\lambda & \mu & \nu & 0
\end{array}\right)=2,
\end{gathered}
$$

then any nonzero finite-dimensional $\mathfrak{h}$-module contains only constants.

An interesting case is the solvable Lie algebra $\mathfrak{h}^{\alpha}=\{p, q$, $x p+\alpha y q\}$, which appears as type 5 in Table 1 . A polynomial is called $\alpha$-homogeneous if the vector field $x p+\alpha y q$ takes it into a multiple of itself. A polynomial module is called $\alpha$-homogeneous if it is spanned by a basis of $\alpha$-homogeneous polynomials. The structure of the $\mathfrak{h}^{\alpha}$-modules depends critically on whether $\alpha$ is rational or irrational (a fact that may be related to the existence of rational Casimir operators, [19]).

Proposition 9. Any $\mathfrak{h}^{\alpha}$-module $(\alpha \neq 0)$ is an $\alpha$-homogeneous polynomial translation module. Moreover, if $\alpha$ is not a positive rational number, then the module is a monomial translation module.

For example, $\mathfrak{h}^{2}=\{p, q, x p+2 y q\}$, and has a nonmonomial module spanned by $x^{4}+x^{2} y, 4 x^{3}+2 x y, x^{2}, x, y, 1$.

The other cases are handled by the following series of propositions, which, for reasons of space, are stated without proof. 
Proposition 10. Every finite-dimensional module for the Lie algebra of type 13 is a direct sum of finitely many of the irreducible modules $m^{(n)}$ spanned by the functions

$$
\begin{aligned}
& (x-y)^{k-n} Q_{n}^{k}\left(\frac{x+y}{x-y}\right), \\
& \quad \text { where } Q_{n}^{k}(z)=\frac{d^{2 n-k}}{d z^{2 n-k}}\left(z^{2}-1\right)^{n}, k=0, \ldots, 2 n .
\end{aligned}
$$

We note that, up to a multiple $Q_{n}^{k}$ is same as the Gegenbauer (ultraspherical) polynomial $C_{k}^{n-k+(1 / 2)}$, and, in particular, $Q_{n}^{n}$ is a multiple of the $n^{\text {th }}$ Legendre polynomial. Indeed, this identification is equivalent to the curious polynomial identity

$$
\frac{d^{k}}{d z^{k}}\left(z^{2}-1\right)^{n}=\frac{k !}{(2 n-k) !}\left(z^{2}-1\right)^{n-k} \frac{d^{2 n-k}}{d z^{2 n-k}}\left(z^{2}-1\right)^{n}, 0 \leq k \leq 2 n
$$

Proposition 11. Every finite-dimensional module for the Lie algebra of type 12 is a direct sum of finitely many of the irreducible modules $m^{(n)}$ spanned by the monomials $x^{i} y^{n}, 0 \leq i \leq n$.

Proposition 12. Any finite dimensional module for the Lie algebra of type 18 is a direct sum of submodules $m_{\mu}=\hat{m}_{\mu} e^{\mu x}$, where $\hat{m}_{\mu}$ is a polynomial $x$-translation module, with $x^{k} \partial_{y} \hat{m}_{\mu} \subset \hat{m}_{\lambda+\mu}$ for $\lambda \in \Lambda$, $k \leq r_{\lambda}$.

Proposition 13. Any finite-dimensional module $m$ for the Lie algebra of type 20 is spanned by polynomials. Moreover, if $\alpha \leq r$, or $\alpha \notin \mathbb{Q}$, then $m$ is spanned by monomials.

With these results in hand, we complete the classification of finitedimensional modules for all the Lie algebras of vector fields from Table 1. In Table 2, the first column shows the Lie algebra considered from Table 1 . The second column indicates whether the module is necessarily spanned by monomials, i.e. single terms of the indicated form. Here $i$, $j, k, n$ are all nonnegative integers. The third column indicates a typical term in any generator of the module. If the module is not spanned by monomials, then the generators will be certain linear combinations of the indicated monomials. The fourth column either indicates ranges of indices which must be included, or, in the case of an arrow, indicates 
other indices which must be included if the given one is. For instance, in case 19 , if the monomial $x^{i} y^{j} e^{\mu x}$ appears in the module, so must $x^{i-1} y^{j} e^{\mu x}$ and $x^{i+r_{\lambda}} y^{j-1} e^{(\mu+\lambda) y}$ (provided $i>0$ and/or $j>0$ ) for each exponent $\lambda$ in the Lie algebra. In all cases, the arbitrary functions (e.g. the $g(y)$ in cases 1-3) or the exponents (e.g. the $\lambda$ and $\mu$ in case 4) all are restricted to belong to a finite set, so that the module is finitedimensional. We note that in all nonmonomial cases, the generators can still be taken to be "exponentially homogeneous," i.e. only one exponential appears. Cases when the module is not generated by monomials must be treated with care, as certain coefficients can also appear.

4. Calculation of cohomology. The final step is the computation of the cohomology spaces $H^{1}\left(\mathfrak{h}, C^{\infty}(M) / m\right)$ for each of the Lie algebras and corresponding modules appearing in Tables 1 and 2 . The results are displayed in Table 3. The first column indicates the dimension of the cohomology space, and the second column gives a representative cocycle $\mathbf{F}$ of each nontrivial cohomology class. Only the vector fields which are actually modified are indicated, i.e. in the notation of (2.3), only the differential operators $\mathbf{v}_{i}+f_{i}$ with nonzero $f_{i}=\left\langle\mathbf{F} ; \mathbf{v}_{i}\right\rangle \neq 0$ are explicitly written down. Again, in the interests of brevity, we will not give the details of most of the calculations. We explain the first four cases for illustrating the basic techniques, and finally discuss the two most complicated cases, types 16 and 18 .

Our computations are considerably simplified by using the following "normalization" procedure. Suppose $\mathfrak{g} \subset \mathfrak{h}$ are two Lie algebras of vector fields. Any $\mathfrak{h}$-module $\boldsymbol{m}$ is automatically an $\mathfrak{g}$-module, and any $\mathfrak{h}$-cocycle $\mathbf{F}$ reduces to an $\mathfrak{B}$-cocycle, denoted by $\left.\mathbf{F}\right|_{\mathfrak{g}}$. Suppose the restricted cocycle $\left.\mathbf{F}\right|_{\mathfrak{S}}$ is cohomologous to an $\mathfrak{B}$-cocycle $\tilde{\mathbf{F}}_{0} \in Z^{1}\left(\mathfrak{B}, C^{\infty}(M) /\right.$ $m$ ) so that, when applied to vector fields in $\mathfrak{B}$, we have $\left.\mathbf{F}\right|_{\mathscr{S}}=\tilde{\mathbf{F}}_{0}+\delta \psi$ for some function $\psi$. On the larger algebra $\mathfrak{h}$, then, the cohomologous cocycle $\tilde{\mathbf{F}}=\mathbf{F}+\delta \psi$ will restrict to the $\mathfrak{S}$-cohomologous cocycle $\tilde{\mathbf{F}}_{0}=$ $\left.\tilde{\mathbf{F}}\right|_{\xi}$. In other words, if a cocycle is cohomologous to a simpler cocycle when restricted to a subalgebra, then it is cohomologous, on the larger algebra, to a cocycle which agrees with the simpler one on the subalgebra. In particular, if we can reduce certain components of $\mathbf{F}$ to zero in the computation of the $\mathfrak{S}$-cohomology class of its restriction $\left.\mathbf{F}\right|_{\mathfrak{s}}$, then the same components can be reduced to zero in the computation of its $\mathfrak{h}$-cohomology class. In this context, we will refer to such a cocycle as normalized. Once we have normalized our cocycle according to a sub- 
algebra $\mathfrak{S}$ of the Lie algebra $\mathfrak{h}$, we can still modify it by coboundaries, but only ones that respect the normalization. Thus, a normalized coboundary must satisfy $\left.\delta \psi\right|_{s}=0$, or equivalently, $\langle\delta \psi ; \mathbf{v}\rangle=\mathbf{v}(\psi) \in m$ for all $\mathbf{v} \in \mathfrak{G}$. This rather trivial remark will serve to enormously simplify cohomology calculations.

Case 1. In the case of the Lie algebra spanned by the single translation vector field $p$, any 1-cocycle is determined by a single function $\langle\mathbf{F} ; p\rangle=f(x, y) \in C^{\infty}(M) / m$. The coboundaries are given by $\langle\delta \psi ; p\rangle=$ $\psi_{x}$. Therefore, by setting $\psi=\int f d x$ to be any integral of $f$, we deduce that any cocycle is a coboundary, and so there is no cohomology.

Case 2. For the Lie algebra generated by $p, x p$, a cocycle $\mathbf{F}$ will be specified by two functions: $\langle\mathbf{F}, p\rangle=f(x, y),\langle\mathbf{F} ; x p\rangle=g(x, y)$. According to case 1 , the cohomology for the subalgebra spanned by $p$ is trivial, hence we can normalize our cocycle so that it automatically vanishes on the basis vector field $p$. To maintain this normalization, then, we are only allowed to modify the cocycle by addition of coboundaries $\delta \psi$ which are trivial on $p$, i.e. satisfy $\langle\delta \psi ; p\rangle=\psi_{x} \in m$. Thus our cocycle (now called F) is determined by a single function $\langle\mathbf{F} ; x p\rangle=$ $g(x, y) \in C^{\infty}(M) / m$. The condition that $\mathbf{F}$ be a cocycle is $\langle\delta \mathbf{F} ; p, x p\rangle=$ $g_{x} \in m$. Since $\langle\delta \psi ; x p\rangle=x \psi_{x}$, two such cocycles are cohomologous if and only if $\tilde{g}=g+x \psi_{x}$, where $\psi \in C^{\infty}(M)$ satisfies the normalization restriction $\psi_{x} \in m$. Since $m$ is generated by monomials $x^{i} h(y), 0 \leq i \leq$ $n_{h}$, the function $g$ must be a linear combination of monomials $x^{j} h(y)$, $0 \leq j \leq n_{h}+1$. (If $h(y)$ is not in $m$, then $n_{h} \equiv-1$, i.e. we can include arbitrary functions of $y$ in $g$.) On the other hand, if $1 \leq j \leq n_{j}+1$, then the monomial $x^{j} h(y)$ can be incorporated into a normalized coboundary, namely $\delta \psi$ where $\psi(x, y)=x^{i} h(y) / j$. Therefore the only contributions to cohomology are functions $g=g(y)$ which do not lie in $m$, and every nonzero cohomology class has as representative $p, x p+$ $g(y)$, where $g \in C^{\infty}(M) / m$.

Case 3. For the Lie algebra of type 3, we normalize using the subalgebra generated by $p, x p$ of case 2 , so that our cocycle satisfies $\langle\mathbf{F} ; p\rangle=0,\langle\mathbf{F} ; x p\rangle=g(y),\left\langle\mathbf{F} ; x^{2} p\right\rangle=h(x, y)$. Normalized coboundaries must satisfy $\langle\delta \psi ; p\rangle=\psi_{x} \in m,\langle\delta \psi ; x p\rangle=x \psi_{x} \in m$. But $m$ contains only functions of $y$, so there are no nonzero coboundaries which obey the normalization restrictions. The conditions that $\mathbf{F}$ define a cocycle are $\left\langle\delta \mathbf{F} ; p, x^{2} p\right\rangle=h_{x}-2 g \in m,\left\langle\delta \mathbf{F} ; x p, x^{2} p\right\rangle=x h_{x}-h \in m$. We 
deduce that $h(x, y)=2 x[g(y)+k(y)]+\tilde{h}(y)$, where $k, \bar{h} \in m$. But $\tilde{h}$ is trivial, so, replacing $g$ by $g+k$, we find a general cohomology representative to be $p, x p+g(y), x^{2} p+2 x g(y)$.

Case 4. For the Lie algebra $\{p, q\}$ of translations in the plane, we normalize the cocycle $\mathbf{F}$ so that $\langle\mathbf{F} ; p\rangle=0$, and hence $\mathbf{F}$ is uniquely determined by a single function $\langle\mathbf{F} ; q\rangle=g(x, y) \in C^{\infty}(M) / m$. The only cocycle condition is $\langle\delta \mathbf{F} ; p, q\rangle=g_{x} \in m$. Note that any function $g_{0}(y)$ $\in C^{\infty}(y)$ can be readily absorbed by a coboundary, so we may assume that $g(0, y)=0$, and the derivative $g_{x}$ uniquely determines $g$. Define Div $m \equiv \partial_{x} m+\partial_{y} m=\left\{\alpha_{x}+\beta_{y} \mid \alpha, \beta \in m\right\}$. We can then write $g_{x}=$ $\varphi+\alpha_{x}+\beta_{y}$ where $\alpha, \beta \in m$, and where $\varphi \in m$ is a representative of an element of the quotient vector space $m / \operatorname{Div} m$. Let $\psi=\int \beta d x$, so that $\psi$ satisfies the normalization restriction $\psi_{x} \in m$. Then the modified function $\tilde{g}=g-\alpha-\psi_{y}$ determines a cohomologous cocycle, and also satisfies $\tilde{g}_{x}=\varphi$. We therefore conclude that the cohomology classes are classified by elements $\varphi$ of the finite-dimensional vector space $m / D i v ~ m$. We can make one further simplification. According to Proposition 6, the module $m$ is a direct sum of submodules $m_{\lambda, \mu}=\hat{m}_{\lambda, \mu} e^{\lambda x+\mu y}$, where each $\hat{\boldsymbol{m}}_{\lambda, \mu}$ is a polynomial translation module. Lemma 14 (stated below), with $\kappa=0$, implies that $m / \mathrm{Div} m \cong m_{0,0} / \mathrm{Div} m_{0,0}$, and we need only look at polynomials for cohomology representatives. In the special case when $m_{0,0}$ is a monomial translation module, spanned by monomials $x^{i} y^{j}$ corresponding to $(i, j)$ in a diagram $\mathbf{S}$, then it is easy to see that the basis elements of $m_{0,0} / \mathrm{Div} m_{0,0}$ will correspond to monomials with $(i, j)$ on an outside corner of $\mathbf{S}$, where by definition, $(i, j) \in \mathbf{S}$ is an outside corner if both $(i+1, j)$ and $(i, j+1)$ do not belong to $\mathbf{S}$.

Lemma 14. Suppose $m=\hat{m} e^{\lambda x+\mu y}$, where $\hat{m}$ is a polynomial translation module. Suppose $\kappa \neq \lambda$. Given $\varphi \in m$ there exists $\psi \in m$ such that $\psi_{x}-\kappa \psi=\varphi$.

Case 16. We may assume without loss of generality that the first vector field is just $q$, i.e. $\xi_{1}(x)=1$, and normalize the cocycle $\mathbf{F}$ so that it vanishes on $q$. Note that the operators $\xi_{k}(x) q+f_{0}^{k}(x)$, with $f_{0}^{k} \in C^{\infty}(x)$, $f_{0}^{k} \notin m$, will certainly contribute to the cohomology, so $H^{1}\left(\mathfrak{h}, C^{\infty}(M) /\right.$ $m)$ contains the nontrivial infinite-dimensional component $\left(C^{\infty}(x) / m_{0}\right)^{r-1}$ $\cong\left(C^{\infty}(x) / m_{0}\right) \otimes V$, where $m_{0}=m \cap C^{\infty}(x)$. Here $V$ denotes a complex vector space of dimension $r-1$ with basis $e_{2}, \ldots, e_{r}$. The remaining cohomology can be computed from that of an associated algebraic complex, and will be finite-dimensional. Define $\partial_{y} m=\left\{f_{y} \mid f \in m\right\}$, $\delta m=$ 
$\operatorname{Span}\left\{\xi_{i} \cdot f \mid f \in m, 1 \leq i \leq r\right\}$. Note that $\partial_{y} \delta m \subset m$. Define $\xi=$ $\Sigma_{k \geq 2} \xi_{k}(x) e_{k} \in C^{\infty}(x) \otimes V \subset C^{\infty}(M) \otimes V$. Then the "algebraic" differential

$$
\delta^{*}: C^{\infty}(M) \otimes \wedge^{k} V \rightarrow C^{\infty}(M) \otimes \wedge^{k+1} V, \delta^{*}(\omega)=\xi \wedge \omega,
$$

defines a complex on the space of $C^{\infty}(M)$-valued $k$-forms on $V$. Consider the quotient complex

$$
\partial_{y} m / \partial_{y}^{2} m \stackrel{\delta_{0}^{*}}{\rightarrow}\left(m / \partial_{y} m\right) \otimes V \stackrel{\delta_{1}^{*}}{\rightarrow}(\delta m / m) \otimes \wedge^{2} V
$$

where $\delta_{1}^{*}, \delta_{0}^{*}$ are the appropriate restrictions of $\delta^{*}$. Let $H_{*}^{1}=\operatorname{Ker} \delta_{1}^{*} /$ $\operatorname{Im} \delta_{0}^{*}$.

THeOREM 15. For a Lie algebra of type 16, with finite dimensional module $m$,

$$
H^{1}\left(\mathfrak{h}, C^{\infty}(M) / m\right) \cong\left\{\left(C^{\infty}(x) / m_{0}\right) \otimes V\right\} \oplus H_{*}^{1}
$$

Proof. Let $\mathbf{F}$ be a normalized cocycle, so $\langle\mathbf{F} ; q\rangle=0$, $\left\langle\mathbf{F} ; \xi_{k}(x) q\right\rangle=$ $f^{k}(x, y), k \geq 2$, and, by the above remarks, we may assume $f^{k}(x, 0)=$ 0 . The cocycle conditions require

$$
\left\langle\delta \mathbf{F} ; q, \xi_{k} q\right\rangle=f_{y}^{k} \in m,\left\langle\delta \mathbf{F} ; \xi_{k} q, \xi_{l} q\right\rangle=\xi_{k} f_{y}^{l}-\xi_{l} f_{y}^{k} \in m
$$

We represent $\mathbf{F}$ by the one-form $\omega_{\mathbf{F}}=\Sigma_{k \geq 2} f_{y}^{k} e_{k} \in m \otimes V$. Since $\mathbf{F}$ is trivial if and only if $f_{y}^{k} \in \partial_{y} m$, we can identify a nontrivial cocycle $\mathbf{F}$ with a nonzero element of the quotient module $\left(m / \partial_{y} m\right) \otimes V$. Furthermore,

$$
\delta^{*}\left(\omega_{\mathrm{F}}\right)=\xi \wedge \omega_{\mathrm{F}}=\sum_{k<l}\left(\xi_{k} f_{y}^{l}-\xi_{l} f_{y}^{k}\right) e_{k} \wedge e_{l} \in \delta m \otimes \wedge^{2} V,
$$

so the cocycle conditions (4.3) are equivalent to the condition $\delta^{*}(\omega) \in$ $m \otimes \wedge^{2} V$, which is the same as requiring that $\delta^{*}(\omega)=0$ as an element of $C^{\infty}(M) / m \otimes \wedge^{2} V$. A function $\psi \in C^{\infty}(M)$ determines a normalized coboundary $\delta \psi$ if and only if $\psi_{y} \in m$. Let $\chi=\psi_{y y}$, so that the one-form corresponding to the coboundary $\delta \psi$ is

$$
\omega_{\delta \psi}=\delta^{*}(\chi)=\Sigma_{k \geq 2} \xi_{k}(x) \chi(x, y) e_{k} .
$$


So $\mathbf{F}=\tilde{\mathbf{F}}+\delta \psi$ are cohomologous if and only if $\omega_{\mathbf{F}}=\omega_{\tilde{F}}+\delta^{*}(\chi)$ are algebraically cohomologous for some $\chi \in \partial_{y} m$. Finally, if $\psi_{y} \in \partial_{y} m$ (hence $\left.\chi \in \partial_{y}^{2} m\right)$ then $\omega_{\delta \psi} \in \partial_{y} m \otimes V$, and is trivial as an element of $\left(m / \partial_{y} m\right)$ $\otimes V$.

For example, in the special case $r=2$, the space $V$ is one-dimensional, and hence $\wedge^{2} V=\{0\}$. Thus (4.2) degenerates to the complex $\partial_{y} m / \partial_{y}^{2} m \rightarrow m / \partial_{y} m \rightarrow 0$, where the first map is just multiplication by $\xi_{2}(x)$. We conclude that, in this particular case, $H^{1}\left(\mathfrak{h}, C^{\infty}(M) / m\right) \cong\left(C^{\infty}(x) /\right.$ $\left.m_{0}\right) \oplus\left(m / \xi_{2} \partial_{y} m\right)$.

If $m=\bigoplus m_{n}$, where $m_{n}=\hat{m}_{n} y^{n}$, is generated by "monomials" $g(x) y^{n}$, with $g \in \hat{m}_{n} \subset C^{\infty}(x)$, it is possible to further analyze the algebraic complex. Define

$$
\delta \hat{\boldsymbol{m}}_{n} \equiv \operatorname{Span}\left\{\xi_{k}(x) g(x) \mid g \in \hat{\boldsymbol{m}}_{n}, k=1, \ldots, r\right\},
$$

so that $m$ is a module if and only if $\delta \hat{m}_{n} \subset \hat{\boldsymbol{m}}_{n-1}, n \geq 1$. Further define the quotient modules $q_{n}=\hat{m}_{n} / \hat{m}_{n+1}, \delta q_{n}=\delta \hat{m}_{n} / \hat{m}_{n}$, so $m / \partial_{y} m \cong \bigoplus_{q_{n}} y^{n}$. The complex (4.2) then decomposes into the direct sum of simpler subcomplexes

$$
q_{n+1} \stackrel{\delta_{0, n}^{*}}{\rightarrow} q_{n} \otimes V \stackrel{\delta_{i, n}}{\rightarrow} \delta q_{n} \otimes \wedge^{2} V
$$

whose cohomology $H_{*, n}^{1}=\operatorname{Ker} \delta_{1, n}^{*} / \operatorname{Im} \delta_{0, n}^{*}$ is readily computable. The full cohomology $H_{*}^{1}$ is the direct sum of these. If $\Sigma h_{i}(x) e_{i}$ is a nontrivial cohomology representative in $H_{*, n}^{1}$, then the corresponding cocycle representing a nontrivial cohomology class in $H^{1}\left(\mathfrak{h}, C^{\infty}(M) / m\right)$ is given by $\langle\mathbf{F} ; q\rangle=0,\left\langle\mathbf{F} ; \xi_{i} q\right\rangle=h_{i}(x) y^{n+1}, 1=2, \ldots, r$.

Case 18. The cohomology for case 18 , which is always finitedimensional, is the most complicated of all. If the module is spanned by monomials, we obtain fairly explicit results using a convenient graph theoretic interpretation; in the non-monomial case, we can only provide partially explicit answers, and bounds on the dimension of the cohomology based on the monomial calculations. We begin by assuming that each submodule $m_{\mu}=\hat{m}_{\mu} e^{\mu x}$ is generated by monomials $x^{i} y^{j} e^{\mu x}$, for $(i, j) \in \mathbf{S}_{\mu} \subset \mathbb{Z}^{+} \times \mathbb{Z}^{+}$. For any pair of integers $(i, j) \in \mathbb{Z}^{2}$ (both positive and negative), define the index

$$
\epsilon_{i, j}^{\mu}= \begin{cases}1, & i \geq 0, j \geq 0,(i, j) \notin \mathbf{S}_{\mu}, \\ 0, & \text { otherwise. }\end{cases}
$$


Normalize so $\langle\mathbf{F} ; p\rangle=0$. Let $\left\langle\mathbf{F} ; x^{k} e^{\lambda x} q\right\rangle=g^{\lambda, k}(x, y) e^{\lambda x}, \lambda \in \Lambda$, $0 \leq k \leq r_{\lambda}$. The first set of cocycle conditions are $\left\langle\delta \mathbf{F} ; p, x^{k} e^{\lambda x} q\right\rangle=$ $g_{x}^{\lambda, k}-k g^{\lambda, k-1} \in \hat{m}_{\lambda}$. An easy induction using Lemma 14 shows that only polynomial functions $g^{\lambda, k}$ will contribute to the cohomology. Integrating these conditions, we deduce that, up to elements of $\hat{m}_{\lambda}$,

$$
g^{\lambda, k}(x, y)=\sum_{i, j} \frac{k !\left(i+r_{\lambda}\right) !}{(i+k) ! r_{\lambda} !} \epsilon_{i+k, j}^{\lambda} c_{i, j}^{\lambda} x^{i+k} y^{j}
$$

where the $c_{i, j}^{\lambda}$ are constants, and the sum ranges over all $(i, j) \in \mathbb{Z}^{2}$ such that $j \geq 0,\left(i+r_{\lambda}, j\right) \notin \mathbf{S}_{\lambda}$, and either $-r_{\lambda} \leq i \leq 0$ or $(i-1, j) \in \mathbf{S}_{\lambda}$. The index $\epsilon_{i+k, j}^{\lambda}$ is zero when the "rational monomial" $x^{i+k} y^{j}$ is either in $m$, or not a polynomial. Furthermore,

$$
\left\langle\delta \mathbf{F} ; x^{k} e^{\lambda x} q, x^{l} e^{\mu x} q\right\rangle=\partial_{y}\left(x^{k} g^{\mu, l}-x^{l} g^{\lambda, k}\right) \in m_{\lambda+\mu} .
$$

Consider first the case $\lambda=\mu$ in (4.5). If $r_{\lambda}=0$, this imposes no restrictions on $g^{\lambda, 0}$. If $r_{\lambda}>0$, and $i=0$, then all the coefficients in (4.4) are 1 or 0 , and (4.5) also imposes no conditions. For $r_{\lambda}>0, i \neq 0$, suppose $k=r_{\lambda}, l=r_{\lambda}-1$. Then (4.5) implies that $c_{i, j}^{\lambda}=0$ unless either $j=0$ or $\left(i+2 r_{\lambda}-1, j-1\right) \in \mathbf{S}_{2 \lambda}$, i.e. unless $\epsilon_{i+2 r_{\lambda}-1, j-1}^{2 \lambda}=0$. This condition ensures that for $\lambda=\mu$ all the other cocycle conditions (4.5) are automatically satisfied. On the other hand, if $r_{\lambda}=0$, then $(i-1$, $j) \in \mathbf{S}_{\lambda}$, so $\left(i+2 r_{\lambda}-1, j-1\right)=(i-1, j-1) \in \mathbf{S}_{2 \lambda}$ automatically. We conclude that $c_{i, j}^{\lambda}=0$ unless $\lambda \in \Lambda_{i, j}$, where

$$
\Lambda_{i, j}= \begin{cases}\left\{\lambda \in \Lambda \mid \epsilon_{i-1, j}^{\lambda}=0, \epsilon_{i+2 r_{\lambda}-1, j-1}^{2 \lambda}=0\right\}, & 0 \neq i \geq-r_{\lambda}, j \geq 0, \\ \Lambda, & i=0, j \geq 0,\end{cases}
$$

Moreover, unless $\lambda \in \Lambda_{i, j}^{+} \equiv\left\{\lambda \in \Lambda_{i, j} \mid \epsilon_{i+r_{\lambda}, j}^{\lambda} \neq 0\right\}$, all the terms in (4.4) involving $c_{i, j}^{\lambda}$ will lie in $\hat{m}_{\lambda}$, and hence will not contribute directly to any cohomology. Note that there are a finite number of integer pairs $(i, j)$ for which the set $\Lambda_{i, j}^{+}$is not empty. Next we analyze (4.5) for $\lambda \neq \mu$. Setting $k=r_{\lambda}, l=r_{\mu}$, we see that either $\epsilon_{i+r_{\lambda}+r_{\mu}, j-1}^{\lambda+\mu}=0$, or $c_{i, j}^{\lambda}=c_{i, j}^{\mu}$. Moreover, if either $r_{\lambda}$ or $r_{\mu}$ is positive, then setting $k=r_{\lambda}-1, l=r_{\mu}$, (or, if $r_{\lambda}=0$, setting $k=r_{\lambda}, l=r_{\mu}-1$ ) we find that either $i=0$, or $\epsilon_{i+r_{\lambda}+r_{\mu}-1, j-1}^{\lambda+\mu}=0$, or $c_{i, j}^{\lambda}=c_{i, j}^{\mu}=0$. Moreover, if these conditions hold, then all the remaining cocycle conditions (4.5) hold trivially. This com- 
pletes the cocycle conditions and the characterization of the cohomology.

These results can be most readily visualized using an elementary graphical approach. For each $(i, j)$ with $i \geq-r_{\lambda}, j \geq 0$, we define a graph $G_{i, j}$ whose vertices correspond to the exponents $\lambda \in \Lambda_{i, j}$. We say that two such exponents are linked if $\epsilon_{i+r_{\lambda}+r_{\mu}, j-1}^{\lambda} \neq 0$ and in this case $G_{i, j}$ contains an edge connecting the vertices corresponding to $\lambda$ and $\mu$. Note that if $j=0$, then there is no linkage, and each graph consists of a single vertex. The edge is called a zero link if $i \neq 0$ and $\epsilon_{i+r_{\lambda}+r_{\mu}-1, j-1}^{\lambda+\mu_{1}} \neq$ 0 . This decomposes the graph $G_{i, j}$ into a finite number of disjoint connected subgraphs, denoted $G_{i, j}^{\gamma}$. We say that $G_{i, j}^{\gamma}$ is a positive subgraph if it contains no zero links and also it contains at least one "positive" vertex $\lambda \in \Lambda_{i, j}^{+}$. We define $d_{i, j}$ to be the number of positive subgraphs $G_{i, j}^{\gamma}$. With each subgraph we associate a constant $k_{i, j}^{\gamma}$ which is arbitrary if the graph is positive, but is otherwise zero. Then the above analysis proves that $\mathbf{F}$ is a nontrivial cocycle if and only if the polynomials $g^{\lambda, k}$ have the form (4.4), where the coefficients are given by $c_{i, j}^{\lambda}=k_{i, j}^{\gamma}$ for each $\lambda \in G_{i, j}^{\gamma}$. Note that this implies that $c_{i, j}^{\lambda}$ vanishes unless $\lambda$ belongs to a positive subgraph $G_{i, j}^{\gamma}$. Therefore the dimension of the space of normalized cocycles is equal to the number of independent constants $k_{i, j}^{\gamma}$. This is the same as the total number of positive subgraphs for all indices $(i, j)$, which is also the sum of the $d_{i, j}$ over all possible $(i, j)$. In particular, since by the earlier remark, there are a finite number of positive graphs, this space is finite dimensional.

Finally we need to deal with coboundaries. Clearly $\psi \in C^{\infty}(M)$ will make a contribution if and only if it is a polynomial. The condition $\psi_{x} \in m$ implies that $\psi$ is a sum of monomials $x^{i} y^{j+1}$ with either $i=0$ or $(i-1, j+1) \in \mathbf{S}_{0}$. Define $e_{i, j}$ to be 1 if $i=0$ or $(i-1, j+1) \in \mathbf{S}_{0}$, and 0 otherwise. In this case, the module conditions imply that $\epsilon_{i+r_{\lambda}-1, j}^{\lambda}=0$, hence $\epsilon_{i+r_{\lambda}+r_{\mu}-1, j}^{\lambda+\mu}=0$, for each exponent $\lambda \in \Lambda$. Therefore, in this case, $\delta \psi\left(x^{k} e^{\lambda x} q\right)=(j+1) x^{i+k} y^{j} e^{\lambda x} \in m$ unless $k=r_{\lambda}$. Thus, we can use this coboundary to absorb one of the constants $k_{i, j}^{\gamma}$, and hence eliminate one positive linkage class. We therefore conclude a general formula for the cohomology in the monomial case:

$$
\operatorname{dim} H^{1}\left(\mathfrak{h}, C^{\infty}(M) / m\right)=\sum_{i, j}\left(d_{i, j}-e_{i, j}\right)
$$

The case when the module is not generated by monomials is even more complicated, and we are not able to offer definitive results. How- 
ever, we can relate the cohomology in this case to a "subset" of an associated monomial cohomology. A general finite-dimensional module always breaks up into submodules $m_{\mu}=\hat{m}_{\mu} e^{\mu x}$, but the individual polynomial $x$-translation module $\hat{\boldsymbol{m}}_{\mu}$ may not be spanned by monomials. We order the monomials lexicographically, so $x^{i} y^{j}>x^{i^{\prime}} y^{j^{\prime}}$ if $i>i^{\prime}$ or $i=i^{\prime}$ and $j>j^{\prime}$. Let $f_{i, j}^{\mu}(x, y)=x^{i} y^{j}+\Sigma_{(k, \eta)<(i, j)} c_{i j}^{k l} x^{k} y^{l},(i, j) \in \mathbf{S}_{\mu}$, be a basis for $\hat{m}_{\mu}$ consisting of "monic" polynomials, satisfying $\partial_{x} f_{i, j}^{\mu}=i f_{-1, j}^{\mu}$, $i \geq 0$. Now, the definitions of the $\epsilon$ 's and the graphs proceeds just as in the monomial case with the same collection of diagrams. We claim that the corresponding cocycles for the nonmonomial case are indexed by a subset of the cocycles for the associated monomial case, so, in some sense, the cohomology for the nonmonomial case is a subset of the associated monomial cohomology. In particular, the cohomology is always finite dimensional, bounded by the right hand side of (4.6). The reason for this is that all the cocycle restrictions on $\mathbf{F}$ can be ordered lexicographically, so that, unless the leading monomial of each $g^{\lambda, k}$ satisfies the cocycle condition, the entire polynomial will not satisfy it. However, the cocycle conditions will impose additional constraints on the lower order terms, so that there may be more requirements than in the monomial case. The only case in which we could run into trouble is when $j=0$, so that when we differentiate with respect to $y$ we end up, not with zero as in the monomial case, but with some lower order polynomial. However, there is never any linkage in the $j=0$ graph, so that every exponent $\lambda$ gives rise to a monomial cohomology class, and hence a potential nonmonomial cohomology class. As the cocycle conditions depend very crucially on the lower order terms, there does not seem to be any further statement that can be made in this regards.

This completes our discussion of the cohomology. One final remark is that Tables 1-3 also solve the "embeddability problem": Given a triple $[\mathfrak{h}, m,[\mathbf{F}]]$ corresponding to a Lie algebra $\mathfrak{g}$ of differential operators, when does there exist a larger Lie algebra $\hat{\mathbf{g}} \subset \mathfrak{g}$ corresponding to a triple $[\mathfrak{h}, \hat{m}, 0]$ having trivial cohomology? (In the construction of Lie algebraic potentials, cf. [12], having trivial cohomology is a big advantage, and going to a larger Lie algebra does not affect the construction.) An inspection of the tables reveals that this is always possible in cases $1,2,4,5,6,7,8,14,16,17,18,19,20,21,22$, but that it is not possible to remove the cohomology class in general in cases 3,9 , $10,11,12,13,15,23,24$. 
Note. An expanded version of this paper, including many explicit examples and more detailed proofs, appears in Differential Geometry, Global Analysis and Topology, CMS Conference Proceedings, Vol. 12, Amer. Math. Soc., Providence, R.I., 1991, 51-84. See our paper in J. Phys. A, 24 (1991), 3995-4008 for further applications of these results.

UNIVERSIDAD COMPLUTENSE, SPAIN

MCGILL UNIVERSITY, QUÉBEC, CANADA

UNIVERSITY OF MINNESOTA

\section{REFERENCES}

[1] M. Abramowitz, and I. Stegun, Handbook of Mathematical Functions, National Bureau of Standards Appl. Math. Series, \#55, U.S. Govt. Printing Office, Washington, D.C., 1970.

[2] M. Ackerman and R. Hermann, Sophus Lie's 1880 Transformation Group Paper, Math. Sci. Press, Brookline, Mass., 1975.

[3] Y. Alhassid, J. Engel, and J. Wu, Algebraic approach to the scattering matrix, Phys. Rev. Lett. 53 (1984), 17-20.

[4] V. I. Arnol'd, Geometrical Methods in the Theory of Ordinary Differential Equations, Springer-Verlag, New York, 1983.

[5] L. Bianchi, Lezioni sulla Theoria dei Gruppi Continui Finiti di Transformazioni, Enrico Spoerri, Editore, Pisa, 1918.

[6] J. E. Campbell, Introductory Treatise on Lie's Theory of Finite Continuous Transformation Groups, The Clarendon Press, Oxford, 1903.

[7] É. Cartan, Sur la Structure des Groupes de Transformations Finis et Continus, Thèse, Paris, Nony, $2^{\mathrm{e}}$ edition, Vuibert, 1913, in Oeuvres Complètes, Pt. I, v. 1, GauthierVillars, Paris, 1952, 137-287.

[8] M. Golubitsky, Primitive actions and maximal subgroups of Lie groups, J. Diff. Geom., 7 (1972), 175-191.

[9] A. González-López, N. Kamran, and P. J. Olver, Lie algebras of vector fields in the real plane, Proc. London Math. Soc., (3) 64 (1992), 339-368.

[10] N. Jacobson, Lie Algebras, Interscience Publ. Inc., New York, 1962.

[11] N. Kamran and P. J. Olver, Equivalence of differential operators, SIAM J. Math. Anal., 20 (1989), 1172-1185.

[12] _ and Lie algebras of differential operators and Lie-algebraic potentials, J. Math. Anal. Appl., 145 (1990), 342-356.

[13] R. D. Levine, Lie algebraic approach to molecular structure and dynamics, in Mathematical Frontiers in Computational Chemical Physics, D. G. Truhlar, ed., IMA Volumes in Mathematics and its Applications, Vol. 15, Springer-Verlag, New York, 1988, 245-261. 
[14] S. Lie, Theorie der Transformationsgruppen, Math. Ann., 16 (1880), 441-528; also Gesammelte Abhandlungen, vol. 6, B. G. Teubner, Leipzig, 1927, 1-94; see [2] for an English translation.

[15] _ Theorie der Transformationsgruppen, vol. 3, B. G. Teubner, Leipzig, 1893.

[16] __ Gruppenregister, Gesammelte Abhandlungen, vol. 5, B. G. Teubner, Leipzig, 1924, 767-773.

[17] I. G. Macdonald, Symmetric Functions and Hall Polynomials, Clarendon Press, Oxford, 1979.

[18] W. Miller, Jr., Lie Theory and Special Functions, Academic Press, New York, 1968.

[19] J. Patera, R. T. Sharp, P. Winternitz, and H. Zassenhaus, Invariants of real low dimension Lie algebras, J. Math. Phys., 17 (1976), 986-994.

[20] F. Schwarz, A factorization algorithm for linear ordinary differential equations, International Symposium on Symbolic and Algebraic Computation, Proceedings of the ACM-SIGSAM 1989, ACM Press, New York, 1989, 17-25.

[21] S. Shnider and P. Winternitz, Nonlinear equations with superposition principles and the theory of transitive primitive Lie algebras, Lett. Math. Phys., 8 (1984), 69-78.

[22] A. V. Turbiner, Quasi-exactly solvable problems and sl(2) algebra, Commun. Math. Phys., 118 (1988), 467-474. 


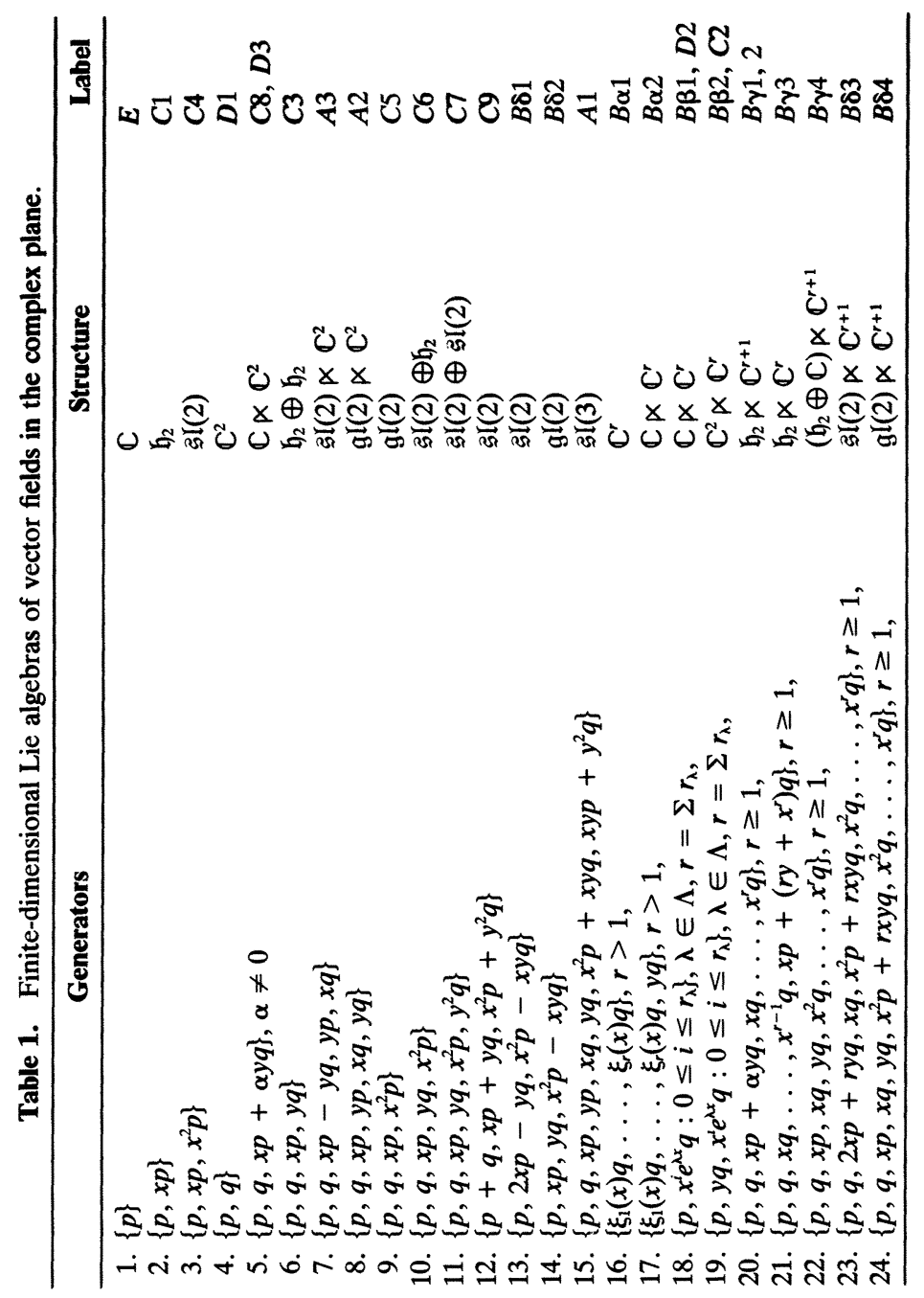




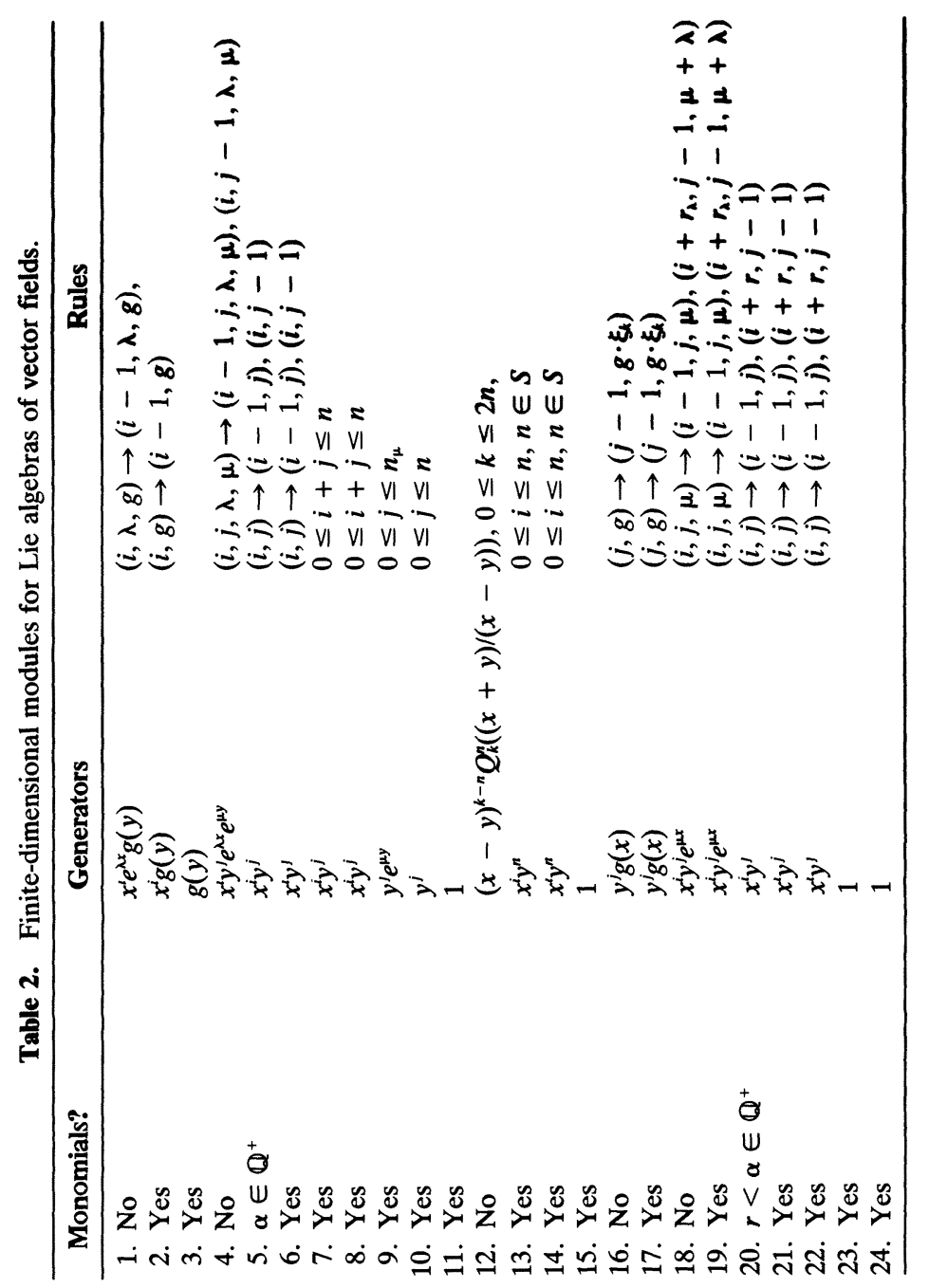




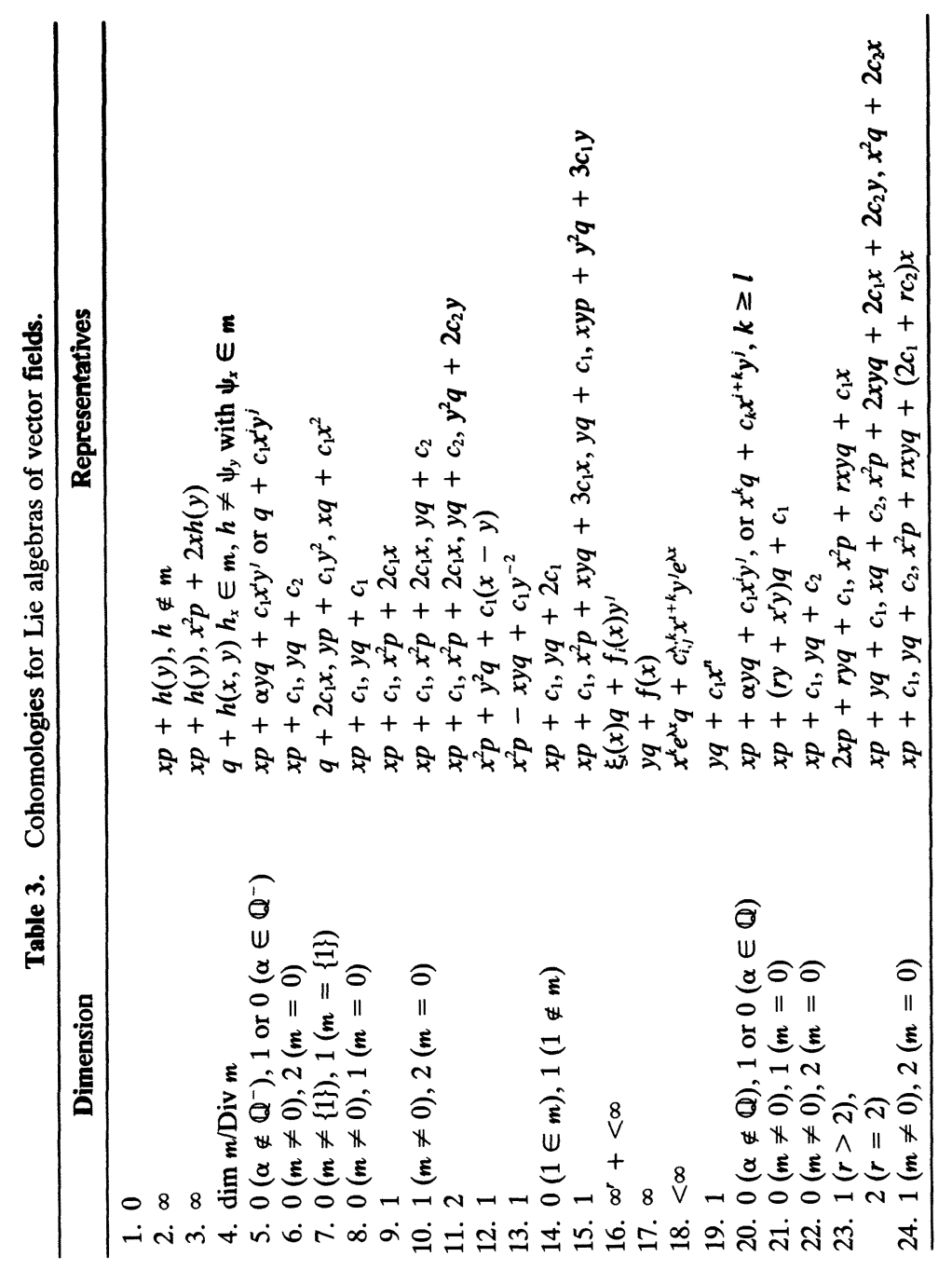

